\title{
Avaliação antagônica in vitro e in vivo de Trichoderma spp. a Rhizopus stolonifer em maracujazeiro amarelo
}

\author{
Marinês Pereira Bomfim; ; Abel Rebouças São José; Tiyoko Nair Hojo Rebouças²; Saulo Sousa de Almeida ${ }^{3}$ Ivan \\ Vilas Boas Souza ${ }^{4}$; Nilma Oliveira Dias ${ }^{4}$.
}

\begin{abstract}
${ }^{1}$ Mestre em Fitotecnia pela Universidade Estadual do Sudoeste da Bahia, Rua Dr. Miguel Alvarenga, 324 apto 21, Botucatu - SP CEP. 18601-090, ${ }^{2}$ Doutores em Fitotecnia - UESB/DFZ - Vitória da Conquista - BA. CEP 45083 900, ${ }^{3}$ Mestre pela Universidade Federal da Bahia - Cruz das Amas - BA, ${ }^{4}$ Mestre em Fitotecnia pela Universidade Estadual do Sudoeste da Bahia, Vitória da Conquista - BA.

Autor para correspondência:Marinês Pereira Bomfim (inesbomfim@fca.unesp.br)

Data de chegada: 13/04/2007. Aceito para publicação em: 15/08/2009.
\end{abstract}

1479

\section{RESUMO}

Bomfim, M.P.; São José, A.R.; Rebouças, T.N.H.; Almeida, S.S.; Souza, I.V.B.; Dias, N.O. Avaliação antagônica in vitro e in vivo de Trichoderma spp. a Rhizopus stolonifer em maracujazeiro amarelo. Summa Phytopathologica, v.36, n.1, p.61-67, 2010.

Para estudar a potencialidade antagônica de espécies de Trichoderma spp. in vitro e in vivo a Rhizopus stolonifer, patógeno causador da podridão floral do maracujazeiro, foram estudadas as espécies de Trichoderma viride, T. virens, T. harzianum e $T$. stromaticum. O crescimento micelial do fitopatógeno foi realizado pelo teste do pareamento de culturas, para crescimento individual foram utilizadas cinco temperaturas. Avaliou-se também o crescimento micelial em $24 \mathrm{~h}$ e $48 \mathrm{~h}$, avaliando a taxa de crescimento dos isolados. Na produção de metabolitos voláteis e não voláteis foram utilizados papel celofane e sobreposição de placas. Em condição de campo os frutos/planta foram tratados com a suspensão na concentração de $2 \times 10^{8}$ Conídios $/ \mathrm{mL}$ sendo avaliado o número médio de frutos aos 15 e 30 . No pareamento de cultura todos os isolados de
Trichoderma spp. apresentaram crescimento micelial, impedindo o desenvolvimento do fitopatógeno, para todos os isolados as temperaturas ideais de crescimento foram de $25^{\circ} \mathrm{C}$ e $30^{\circ} \mathrm{C}$. Nos períodos de incubação de 24 e $48 \mathrm{~h}$, foram constatadas diferenças significativas no crescimento micelial entre os isolados os antagonistas apresentaram velocidade de crescimento maior que o fitopatógeno. Houve uma produção de metabólitos voláteis e não voláteis de ação antifúngica ao $R$. stolonifer. No ensaio em campo houve diferença significativa entre os tratamentos, verificando-se que o melhor resultado entre os antagonistas em estudo cujos percentuais de pegamento foram 74\% para os tratamentos Trichoderma harzianum e T. virens, e os tratamentos T. viride e T. stromaticum obtiveram um porcentual de $75 \%$ enquanto a testemunha obteve um percentual de $42 \%$.

Palavras-chave adicionais: Controle Biológico, Podridão Floral, Manejo Integrado de Praga.

\section{ABSTRACT}

Bomfim, M.P.; São José, A.R.; Rebouças, T.N.H.; Almeida, S.S.; Souza, I.V.B.; Dias, N.O. Antagonic effect in vitro and in vivo of Trichoderma spp. to Rhizopus stolonifer in yellow passion fruit. Summa Phytopathologica, v.36, n.1, p.61-67, 2010.

Floral rot of passion fruit is caused by the fungus Rhizopus stolonifer, which attacks newly open flowers and young little fruits. The infection starts in the inner parts of the flower buds where it causes water-soaked lesions, whose floral tissues acquire a dark-brown coloration. The presence of dark mycelium and fungus fructification is often noticed on the lesions. The present work aimed at studying the in vitro and in vivo antagonistic potentiality of species of Trichoderma spp. to Rhizopus stolonifer. In order to assess the antagonistic efficiency of biological control agents in the floral rot of yellow passion fruit, in vitro and in vivo tests were carried out with these species: Trichoderma viride, T. virens, T. harzianum and $T$. stromaticum, at the Biofactory of the State University of Southwestern Bahia (Universidade Estadual do Sudoeste da Bahia), campus of Vitória da Conquista - BA, and at a farm in the municipality of Eunápolis - BA. In the in vitro tests the mycelial growths of the phytopathogen and antagonists at the temperatures of $10^{\circ} \mathrm{C}, 15^{\circ} \mathrm{C}$, $20^{\circ} \mathrm{C}, 25^{\circ} \mathrm{C}$ and $30^{\circ} \mathrm{C}$ were evaluated as well as the mycelial growth within $24 \mathrm{~h}$ and $48 \mathrm{~h}$, in order to verify the growth rate of the isolates. To inhibit the mycelial growth of the phytopathogen, the pairing of cultures was carried out to assess the potentiality of antagonists and the production of volatile and non-volatile metabolites. Field, the antagonists were assessed according to their capacity. In the of preventing the infection by Rhizopus stolonifer, the average number of effective fruits being evaluated on the $15^{\text {th }}$ and $30^{\text {th }}$ day after the application of antagonists. Through the obtained results, it was noticed that the best temperatures for the growth of antagonists and phytopathogen were at $25^{\circ} \mathrm{C}$ and $30^{\circ} \mathrm{C}$ for all isolates. During the incubation periods of 24 and $48 \mathrm{~h}$, significant differences in the mycelial growth were evidenced among isolates of Trichoderma spp. In relation to $R$. stolonifer, the antagonists demonstrated growth speed significantly higher than the phytopathogen. In paired culture, all the isolates of Trichoderma spp. showed fast mycelial growth, thus preventing the development of the phytopathogen, demonstrating antagonism in the production of volatile and non volatile metabolites with fungal action against $R$. stolonifer. In the field test, there was significant effect in the treatments, a higher fruit set of $74 \%$ was noted for the treatments $\mathrm{T}_{3}$ - Trichoderma harzianum and $\mathrm{T}_{5}-T$. virens, and $75 \%$ for. The treatments $\mathrm{T}_{2}-T$. viride and $\mathrm{T}_{4}-T$. stromaticum the control obtained a lower percentage $42 \%$.

Keywords: Biological control, floral rot, integrated pest management. 
O Brasil é o maior produtor mundial de maracujá-amarelo (Passiflora edulis Sims f. flavicarpa Deg.) com uma produção aproximada de 330.777 toneladas (7). Os mercados de suco e de fruta fresca têm crescido, e a produção, embora estagnada nos últimos anos, é substancialmente maior que aquela de décadas anteriores. $\mathrm{O}$ destino da produção varia de acordo com a região, porém verifica-se ao longo das últimas duas décadas uma inversão: o mercado da fruta fresca que absorvia aproximadamente $30 \%$ da produção nacional, hoje absorve mais da metade (14).

A produtividade dos pomares brasileiros de maracujá é bastante variável, estando a média nacional em torno de 10 a 12T/ha/ano, embora existam pomares com produção acima de 40T/ha/ano em plantações de vida útil de dois anos, sendo baixa quando comparada à de outros países produtores, como por exemplo, o Havaí (EUA) com 45T/ha/ ano (13).

A baixa produtividade dos pomares brasileiros está associada a diversos fatores, sendo as pragas as principais responsáveis pela redução da vida útil de produção.

Dentre as diversas doenças de importância econômica para a cultura do maracujazeiro o Rhizopus stolonifer trata-se de uma enfermidade recente porém os danos causados por este fungo pode chegar em algumas condições especiais a quase $100 \%$ de perda na produção (15).

O fungo Rhizopus se desenvolve com maior intensidade após período chuvoso o que contribui para a queda de flores.

Segundo Pizza Junior (1991) citado por Ataíde (2), essa condição compromete a produção, por impedir a polinização ou o desenvolvimento do grão de pólen. Sob precipitações, a atividade dos insetos polinizadores se reduz ou fica ausente, além de impedir a polinização artificial que e de extrema importância para a cultura, com isso a disseminação da doença aumenta com o emprego dessa prática.

Góes (8) relatou sobre a podridão floral do maracujazeiro, afirmando ser essa doença causada pelo fungo Rhizopus; trata-se de uma enfermidade relativamente nova para a cultura e, por isso, existem poucas informações sobre a sua distribuição geográfica, incidência, níveis de prejuízos e controle. Inicialmente foi verificada na Região de Brasília - Distrito Federal, provocando graves prejuízos na cultura do maracujazeiro. Atualmente tem sido verificada na região Sudeste, especialmente nos estado de São Paulo e Minas Gerais, e na região Norte, em plantios localizados no Pará. Alguns relatos dão conta do seu surgimento nos cultivos de maracujazeiros no Extremo Sul da Bahia.

Rhizopus stolonifer ataca as flores recém abertas e frutinhos novos, a infecção iniciam-se nas partes internas dos botões florais onde causa lesões encharcadas, do tipo podridão mole, cujos tecidos florais adquirem coloração pardo-escuro. Sobre as lesões, frequentemente observa-se a presença de micélio escuro e frutificação do fungo.

Os botões florais afetados tornam-se murchos e caem, deixando apenas as sépalas. Nos botões florais afetados, pode-se observar a presença de micélio do fungo e as respectivas frutificações, as quais aparentam pequenas pontuações de cor preta.

Sob condições de elevada umidade, especialmente após período chuvoso prolongado, micélio e frutificação do fungo podem ser observadas na superfície das flores.

A medida predominante de controle que se tem conhecimento até o momento é a pulverização com fungicida na época da florada. No entanto, algumas dificuldades são encontradas neste tipo de controle. Sabe-se que a severidade da doença aumenta quando períodos longos de chuva ou umidade elevada ocorrem por ocasião do pico do florescimento, o que dificulta o controle com fungicida, a ocorrência de várias floradas aumenta a incidência da doença ficando mais difícil o seu controle (15).

O fungo Rhizopus é de difícil controle através dos fungicidas disponíveis no mercado. Na região do Extremo Sul da Bahia, foram testados mais de 20 fungicidas, visando controlar o fitopatógeno, sem sucesso. Apenas o fungicida tebuconazole tem demonstrado boa eficiência no controle do Rhizopus, entretanto, tem sido observado em condições de campo, tanto no Norte do Espírito Santo, quanto no Extremo Sul da Bahia e platô de Neópolis em Sergipe, que este fungicida, apesar de apresentar um bom controle sobre o fitopatógeno, provoca uma fitotoxicidade bastante pronunciada na planta, que às vezes leva mais de 40 dias para recuperação (15).

Apesar da importância da doença, são poucas as informações acerca da incidência, níveis de prejuízos e controle. O controle biológico tem sido bastante eficiente, nas condições de Eunapólis - BA, (2) onde apesar do número elevado de flores infectadas houve controle do fungo, com percentual de vingamento de $70 \%$ em condições de campo.

O presente trabalho tem por objetivo avaliar o antagônismo de espécies de Trichoderma spp. in vitro e in vivo a Rhizopus stolonifer agente causal da podridão floral do maracujazeiro.

\section{MATERIAL E MÉTODOS}

O presente trabalho foi conduzido na Biofábrica Pesquisa e Produção de Microrganismos da Universidade Estadual do Sudoeste da Bahia, campus de Vitória da Conquista - BA e na propriedade agrícola Água Rosada no município de Eunapólis - BA, onde foram estudados:

\section{Obtenção dos Agentes de Controle Biológico (ACB)}

Os ACB utilizados no experimento foram: Trichoderma viride cepa da Biofábrica - UESB, Trichoderma stromaticum proveniente da CEPLAC - BA, Trichoderma harzianum - da Empresa Itaforte - SP e Trichoderma virens, obtidos na Mata ciliar de Contendas do Sincorá - BA identificado pela UFRPE.

\section{Antagonismo entre Trichoderma spp. e Rhizopus stolonifer em cultivo pareado}

Para avaliar o antagonismo de Trichoderma spp. a $R$. stolonifer, foi feito o teste do pareamento de culturas em BDA. Onde foi colocado em cada placas de Petri, nos quais discos de cultura do antagonistas e do fitopatógeno foram colocados em lados diametralmente opostos. Os discos foram obtidos de culturas puras de ambos os fungos, as quais foram incubadas sob temperatura de $25^{\circ} \mathrm{C}$ e fotoperíodo de $12 \mathrm{~h}$ (15). O potencial de antagonismo dos isolados foi avaliado aos 4, 8, 12 e 16 dias, em cinco repetições, pela medida do crescimento micelial (em $\mathrm{cm}$ ) das colônias de $R$. stolonifer.

Efeito de temperaturas no crescimento micelial de Trichoderma spp. e Rhizopus stolonifer

Foram retirados discos agarizados de $7 \mathrm{~mm}$ de diâmetro, de culturas crescidas por sete dias, dos antagonistas e fitopatógeno, sendo depositados no centro de placas de Petri de $90 \mathrm{~mm}$, separadamente, sendo incubado na ausência de luz nas temperaturas $10^{\circ} \mathrm{C}, 15^{\circ} \mathrm{C}$, $20^{\circ} \mathrm{C}, 25^{\circ} \mathrm{C}$ e $30^{\circ} \mathrm{C}$. Para cada tratamento foram realizadas cinco repetições, sendo cada repetição formada por cinco placas em um delineamento inteiramente casualizado. 
Após cinco dias de incubação, foi avaliado o crescimento da colônia através da medição do diâmetro utilizando-se uma régua milimetrada.

\section{Crescimento de Rhizopus stolonifer e espécies de Trichoderma spp. em cultivo individual}

A metodologia aplicada neste experimento, baseou-se em JackischMatsuura e Menezes (9). Foram determinados o crescimento micelial e a taxa de crescimento dos isolados de Trichoderma spp. e R. stolonifer.

Discos de $7 \mathrm{~mm}$ de diâmetro foram retirados das margens das colônias dos isolados de ambos os fungos com sete dias e transferidos para o centro das placas de Petri contendo BDA. As placas foram mantidas a $25^{\circ} \mathrm{C}$ sob fotoperíodo de $12 \mathrm{~h}$. Para cada tratamento foram feitas cinco repetições, em um delineamento inteiramente casualizado. $\mathrm{O}$ registro do crescimento micelial deu-se pela medida do diâmetro de cada colônia, com auxílio de régua milimetrada, em dois intervalos de tempo, $24 \mathrm{~h}$ e $48 \mathrm{~h}$. A taxa de crescimento de cada organismo foi determinada aplicando-se a fórmula adaptada de Lilly e Barnett (1951), citada por Jackisch-Matsuura e Menezes (9):

$$
\begin{aligned}
& \text { Txc }=\frac{\mathrm{C}_{2}-\mathrm{C}_{1}}{\mathrm{~T}_{2} \mathrm{~T}_{1}} \\
& \text { onde: } \\
& \text { Txc }=\text { taxa de crescimento }(\mathrm{mm} / \mathrm{h}) ; \\
& \mathrm{C}_{2}=\text { crescimento após } 48 \text { horas de incubação; } \\
& \mathrm{C}_{1}=\text { crescimento após } 24 \text { horas de incubação; } \\
& \mathrm{T}_{2}=48 \mathrm{~h} \text { e } \mathrm{T}_{1}=24 \mathrm{~h} .
\end{aligned}
$$

Produção de metabólitos voláteis de Trichoderma spp. sobre o crescimento micelial de Rhizopus stolonifer

Foi estudado o efeito de extratos livres de células de Trichoderma spp. sobre o crescimento micelial de $R$. stolonifer. Placas de Petri contendo meio BDA, foram posicionadas umas sobre as outras, sendo colocado na parte inferior um disco de ágar de $7 \mathrm{~mm}$ de Trichoderma spp. e na superior um disco de $R$. stolonifer. As placas foram vedadas e incubadas a $25^{\circ} \mathrm{C}$, com fotoperíodo de $12 \mathrm{~h}$ por sete dias. Cada tratamento foi composto por cinco repetições em delineamento inteiramente casualizado. Para a testemunha foi utilizado apenas discos de $R$. stolonifer tanto na parte inferior como na superior da placa de Petri.

Metabólitos não voláteis produzidos por Trichoderma spp.

O método consistiu em cobrir assepticamente toda a superfície do meio (BDA), com discos de papel celofane (9 mm de diâmetro). A seguir, discos de ágar contendo micélio mais conídios de Trichoderma spp. foram colocados no centro, sobre a superfície do papel celofane (5). As placas foram incubadas por $48 \mathrm{~h}$ à temperatura ambiente, sob luz contínua. Após as $48 \mathrm{~h}$, as placas com os antagonistas já estabelecidos sobre o papel celofane foram abertas em câmara asséptica, e retirou-se o papel celofane juntamente com as colônias dos antagonistas. As placas foram invertidas e adicionou-se aproximadamente $10 \mathrm{~mL}$ de clorofórmio na sua parte inferior, a fim de eliminar resíduos estruturais dos antagonistas em estudo. Após a evaporação do produto, voltou-se à posição original das placas, onde foram colocados discos de $7 \mathrm{~mm}$ de diâmetro retirados das margens das colônias do fitopatógeno. As placas foram mantidas a $25^{\circ} \mathrm{C}$ sob fotoperíodo de $12 \mathrm{~h}$. A testemunha foi constituída de placas contendo apenas o fitopatógeno sem a presença de metabólitos.

Em delineamento inteiramente casualizado com cinco repetições, os tratamentos foram comparados com base no diâmetro das colônias do fitopatógeno, após este alcançar a borda da placa, o que ocorreu cinco dias após a inoculação.

Interação in vivo

Foram utilizadas 1.250 flores de um pomar comercial de maracujazeiro amarelo (Passiflora edullis Sims f. flavicarpa Deg), com sete meses de idade em período de floração.

Foram estudados cinco tratamentos, $\mathrm{T}_{1}$ - Testemunha; $\mathrm{T}_{2}$ Trichoderma viride; $\mathrm{T}_{3}-$ T. harzianum; $\mathrm{T}_{4}-$ T. stromaticum e $\mathrm{T}_{5}-T$. virens, com cinco repetições sendo 50 flores por repetição em um delineamento em blocos casualizados.

As avaliações foram realizadas aos 15 e 30 dias após a aplicação dos antagonistas, efetuando-se a contagem do número de frutos vingados, a fim de se obter o número médio de frutos efetivos (NMFE), de acordo com Góes (8).

$\mathrm{NMFE}=(\mathrm{A} /(\mathrm{A}+\mathrm{B})) \times 100$

$\mathrm{A}=\mathrm{N}^{\circ}$ DE BOTÕES VINGADOS

$\mathrm{B}=\mathrm{N}^{\circ}$ DE BOTÕES CAÍDOS

\section{RESULTADOS E DISCUSSÃO}

\section{Crescimento micelial em cultivo pareado de Trichoderma spp.} e $R$. stolonifer.

Todos os isolados de Trichoderma spp. apresentaram rápido crescimento micelial em BDA. Com apenas três dias de incubação, os antagonistas já inibiram o desenvolvimento de $R$. stolonifer. Os diâmetros médios das colônias do fitopatógeno, quando pareados com os isolados de Trichoderma spp. diferiram estatisticamente da testemunha.

Pelos dados da Tabela 1, verifica-se que houve diferença significativa entre os tratamentos, onde o $\mathrm{T}_{2}$ apresentou uma maior inibição no crescimento do fitopatógeno, quando comparado aos demais tratamentos. Os tratamentos $\mathrm{T}_{4}$ e $\mathrm{T}_{5}$ proporcionaram uma menor ação antagônica no crescimento do fitopatógeno, em comparação aos outros. A menor ação antagônica nesses tratamentos pode ter resultado de uma maior competição pelos nutrientes do meio, ou uma menor produção de protease e cisteína, enzimas produzidas pelas espécies de Trichoderma que inativam a capacidade enzimatica do fitopatógeno. Ao comparar os tratamentos com a testemunha foi verificado que todos os isolados foram eficientes, visto que a testemunha aos quatro

\begin{tabular}{|c|c|c|c|c|}
\hline \multirow{2}{*}{ Tratamentos } & \multicolumn{4}{|c|}{ Dias de avaliação } \\
\hline & 4 & 8 & 12 & 16 \\
\hline T Rhizopus. stolonifer & $9,00 \mathrm{a}$ & $9,00 \mathrm{a}$ & $9,00 \mathrm{a}$ & $\overline{9,00 \mathrm{a}}$ \\
\hline $\mathrm{T}^{1-}-R$. stolonifer $\mathbf{x}$ T. Viride & ${ }^{(1)} 0,50 \mathrm{~d}$ & $0,66 \mathrm{~d}$ & $1,00 \mathrm{~d}$ & $1,16 \mathrm{~d}$ \\
\hline $\mathrm{T}_{3}^{2}-R$. stolonifer $\mathbf{x}$ T. harzianum & $1,16 \mathrm{c}$ & $1,50 \mathrm{c}$ & $1,66 \mathrm{c}$ & $1,83 \mathrm{c}$ \\
\hline $\mathrm{T}_{4}-R$. stolonifer $\mathbf{x}$ T. Stromaticum & $n 2,00 \mathrm{~b}$ & $2,16 \mathrm{~b}$ & $2,66 \mathrm{~b}$ & $3,00 \mathrm{~b}$ \\
\hline $\mathrm{T}_{5}-R$. stolonifer $\mathbf{x} T$. virens & $2,00 \mathrm{~b}$ & $2,33 \mathrm{~b}$ & $3,00 \mathrm{~b}$ & $3,00 \mathrm{~b}$ \\
\hline
\end{tabular}
dias já cobria $100 \%$ da placa.

Mughogho (1967, 1968), citado por Dennis \& Webster, (4), relatou que Trichoderma viride afetou o crescimento de Rhizoctonia solani e

Tabela 1. Crescimento micelial de Rhizopus stolonifer em presença dos isolados de Trichoderma spp. avaliado por meio da técnica do pareamento. 
Armillaria mellea mais do que qualquer outra espécie testada e que, isolados de T. harzianum não inibiram o crescimento desses fungos, diferentemente dos resultados obtidos neste trabalho, em que essa espécie inibiu o crescimento micelial de $R$. stolonifer. A redução do crescimento da colônia do fitopatógeno, na presença de Trichoderma spp. pode ser atribuída a liberação de metabólitos.

Ainda sobre a ação biocontroladora de espécies de Trichoderma spp. sobre fungos fitopatogênicos (12), em estudos in vitro, em culturas pareadas, constataram a ocorrência de zonas de inibição, com as espécies de Trichoderma harzianum e T. viride em relação ao poatógeno Colletotrichum graminicola. Verificaram, ainda, que essas espécies produziram metabólitos extracelulares, não voláteis, termoestáveis, difusíveis, que inibiram o crescimento do patógeno.

Efeito de diferentes temperaturas no crescimento micelial de Trichoderma spp. e Rhizopus stolonifer

Os resultados podem ser observador na (Figura 1) onde as melhores temperaturas de crescimento dos isolados foram de $25^{\circ} \mathrm{C}$ e $30^{\circ} \mathrm{C}$ para todos os isolados em estudo, não havendo diferença significativa. Nessas temperaturas antagonistas e fitopatógeno cobriram toda a superfície do meio, concordando com o observado por Jaill et al., (10). Estes autores realizando investigações sobre as temperaturas ideais de crescimento de Trichoderma spp. observaram que as temperaturas ótimas de crescimento de Trichoderma spp. foram 25 a $28^{\circ} \mathrm{C}$, e verificaram que nas temperaturas de 10 a $17^{\circ} \mathrm{C}$ os isolados não apresentaram um crescimento micelial satisfatório. No presente estudo, verificou-se também uma inibição no crescimento micelial de todos os isolados nas temperaturas de 10 e $15^{\circ} \mathrm{C}$.

Outros autores estudando o antagonismo de Trichoderma harzianum, T. viride a Gliocladium roseum observaram que os antagonistas não apresentaram crescimento micelial a 0 e $2^{\circ} \mathrm{C}$ e um pequeno desenvolvimento a 5 e $10^{\circ} \mathrm{C}$, o que concorda com as observações do presente trabalho.

No tratamento a $20^{\circ} \mathrm{C}$ houve um razoável crescimento dos isolados, porém, não cobriram toda a superfície do meio, ficando claro que as melhores temperaturas para o desenvolvimento, tanto dos antagonistas quanto do fitopatógeno são acima de $20^{\circ} \mathrm{C}$.

Através dos resultados obtidos, pode-se afirmar que tanto $R$. stolonifer como as espécies de Trichoderma spp. têm a mesma temperatura ótima de crescimento, aspecto importante, já que em estudos de controle biológico, o antagonista deve ter preferencialmente a mesma temperatura de crescimento do fitopatógeno; assim de acordo com Dennis e Webster (4) os isolados de uma mesma espécie, embora sejam morfologicamente semelhantes, podem diferir fisiologicamente e atuarem de maneira diferente quanto ao antagonismo, dependendo, portanto, mais das características fisiológicas do isolado que da espécie propriamente dita. Além da possível variabilidade genética inerente a cada isolado, existem também fatores externos como equilíbrio nutricional e influência de temperatura que afetam o desenvolvimento.

\section{Crescimento micelial em cultivo individual de Trichoderma} spp. e Rhizopus stolonifer.

Foram constatada diferença significativa no crescimento micelial entre os isolados de Trichoderma spp. com relação a $R$. stolonifer, tanto no período de 24 horas como no 48 horas Verificando que todos os isolados apresentaram velocidade de crescimento significativamente maior que o fitopatógeno $(1,250 \mathrm{~mm} / \mathrm{h})$ Tabela 2 .

Esta diferença ocorre pelas características de crescimento das espécies de Trichoderma spp. que possuem um crescimento rápido, geralmente em poucas horas ocupando toda a superfície da placa (3).

Os resultados obtidos estão de acordo com os dados de JackischMatsuura e Menezes (9), ao estudarem o controle de Pythium aphanidermatum em fumo com espécies de Trichoderma. Os autores citados utilizaram, além das referidas espécies, isolados de Trichoderma viride e T. harzianum, os quais apresentaram taxas de crescimento, estatisticamente semelhantes entre si corroborando o presente estudo.

\section{$\Xi 10^{\circ} \mathrm{C} \boxminus 15^{\circ} \mathrm{C}$ 目20ㅇ $\square 25^{\circ} \mathrm{C} \nabla 30^{\circ} \mathrm{C}$}

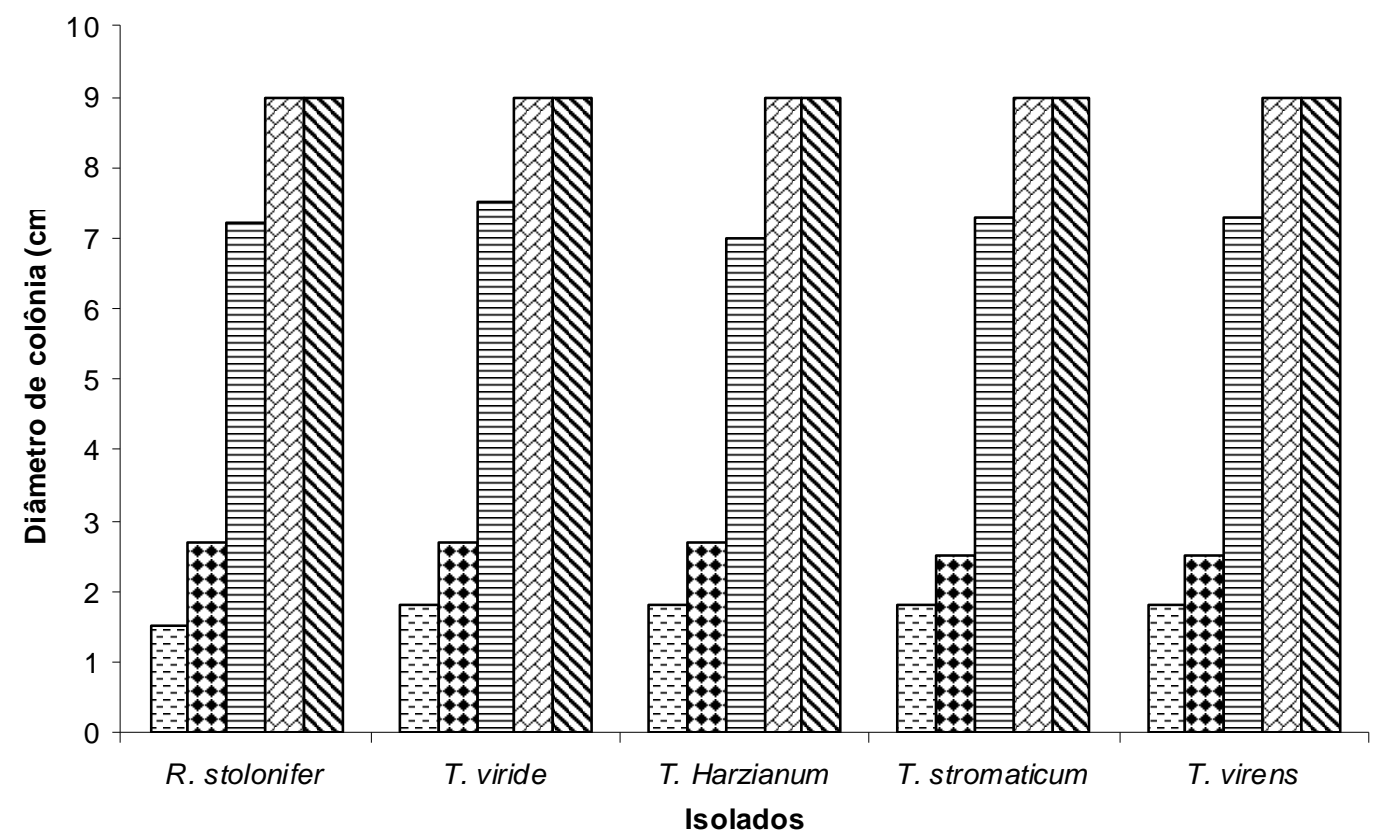

Figura 1. Diferenças entre diâmetro médio de colônias de Trichoderma spp. e R. stolonifer incubado aos cinco dias, sob diferentes temperaturas. Médias seguidas pela mesma letra não diferem entre si pelo teste Tukey a $5 \%$ de probabilidade. 
Tabela 2. Crescimento radial dos isolados de Trichoderma spp. e Rhizopus stolonifer.

\begin{tabular}{|c|c|c|c|}
\hline \multirow{2}{*}{ Tratamentos } & \multicolumn{2}{|c|}{ Crescimento micelial (mm) } & \multirow{2}{*}{$\begin{array}{c}\text { Taxa de } \\
\text { crescimento } \\
(\mathrm{mm} / \mathrm{h})\end{array}$} \\
\hline & 24 horas & 48 horas & \\
\hline $\mathrm{T}$ - Rhizopus stolonifer & $25,00 \mathrm{~b}$ & $55,00 \mathrm{~b}$ & $1,250 \mathrm{~b}$ \\
\hline $\mathrm{T}_{2}-$ Trichoderma viride & 28,70 a & 71,67 a & 1,793 a \\
\hline $\mathrm{T}_{3}-$ Trichoderma harzianum & $28,71 \mathrm{a}$ & 71,63 a & 1,788 a \\
\hline $\mathrm{T}_{4}-$ Trichoderma stromaticum & 28,69 a & 71,67 a & 1,793 a \\
\hline $\mathrm{T}_{5}-$ Trichoderma virens & 28,78 a & 71,66 a & 1,786 a \\
\hline
\end{tabular}

Médias seguidas pela mesma letra na coluna não diferem entre si pelo teste Tukey a 5\% de probabilidade.

Metabólitos voláteis produzidos por Trichoderma spp. sobre o crescimento micelial de Rhizopus stolonifer

Pelo teste de antibiose foi possível verificar a produção de metabólitos voláteis das espécies de Trichoderma spp. havendo uma inibição no desenvolvimento micelial do fitopatógeno. Os tratamentos $\mathrm{T}_{2}$ e $\mathrm{T}_{3}$, produziram notável efeito fungistático sobre o fitopatógeno.

$\mathrm{O}$ tratamento $\mathrm{T}_{2}$ apresentou uma maior produção de metabólitos, visto que sua colônia, ao atingir cerca de $4,0 \mathrm{~cm}$ inibiu completamente o crescimento de $R$. stolonifer (Figura 2).

Os tratamentos $\mathrm{T}_{4}$ e $\mathrm{T}_{5}$ apesar de produzirem metabólitos capazes de inibir o crescimento do fitopatógeno, apresentaram instabilidade metabólica, pois, apesar de apresentar halo de inibição superior à média, após alguns dias, houve a retomada de crescimento de $R$. stolonifer.

O crescimento do fitopatógeno foi reduzido na presença de Trichoderma spp. comparando com a testemunha que aos quatro dias já tomava quase $100 \%$ da placa, o que está de acordo com Dennis \& Webster (4), os quais o afirmaram que espécies de Trichoderma são eficientes produtoras de metabólitos voláteis em meio de cultura. Esses autores explicam que os antibióticos voláteis atuam sobre os fungos suscetíveis através da inibição do crescimento micelial.

Teste de antibiose e efeito de metabólitos não voláteis de Trichoderma spp. na sobrevivência de Rhizopus stolonifer

Observa-se na Figura 3 que os antagonistas produziram efeito fungistático sobre o fitopatógeno, todos os isolados produiram substâncias capazes de inibir o crescimento de $R$. stolonifer, apresentando diferença significativa em relação a testemunha, apesar de não diferirem entre si. Os metabólitos não voláteis produzidos pelos antagonistas provocaram um desenvolvimento micelial menos denso reduzindo o tamanho da colônia do patógeno em comparação com a testemunha. Confirmou-se assim a produção de metabólitos não voláteis pelos antagonistas em estudo.

Os primeiros trabalhos científicos demonstrando a produção de antibióticos por Trichoderma spp. foram realizados por Weidling (17), que conseguiu identificar os antibióticos gliotoxina e viridina. Dennis \& Webster (5) demonstraram que isolados deste gênero foram capazes de produzir metabólitos voláteis e não voláteis, com efeito inibitório sobre o crescimento de vários fungos. Entre os metabólitos voláteis, há gases como: etileno e cianeto de hidrogênio, acetaldeído, acetona, etanol e dióxido de carbono (16), que afetam o crescimento microbiano. Esses gases são ativos a baixas concentrações, mas não são considerados como antibióticos.

Entre os efeitos provocados pelos antibióticos, podem ser observadas a redução ou paralisação do crescimento e esporulação, redução na germinação de esporos, além de distorções na hifa e endólise. Os antibióticos são produtos do metabolismo secundário de seus produtores, e podem ser mais importantes na inibição de outros organismos do que a competição por nutrientes.

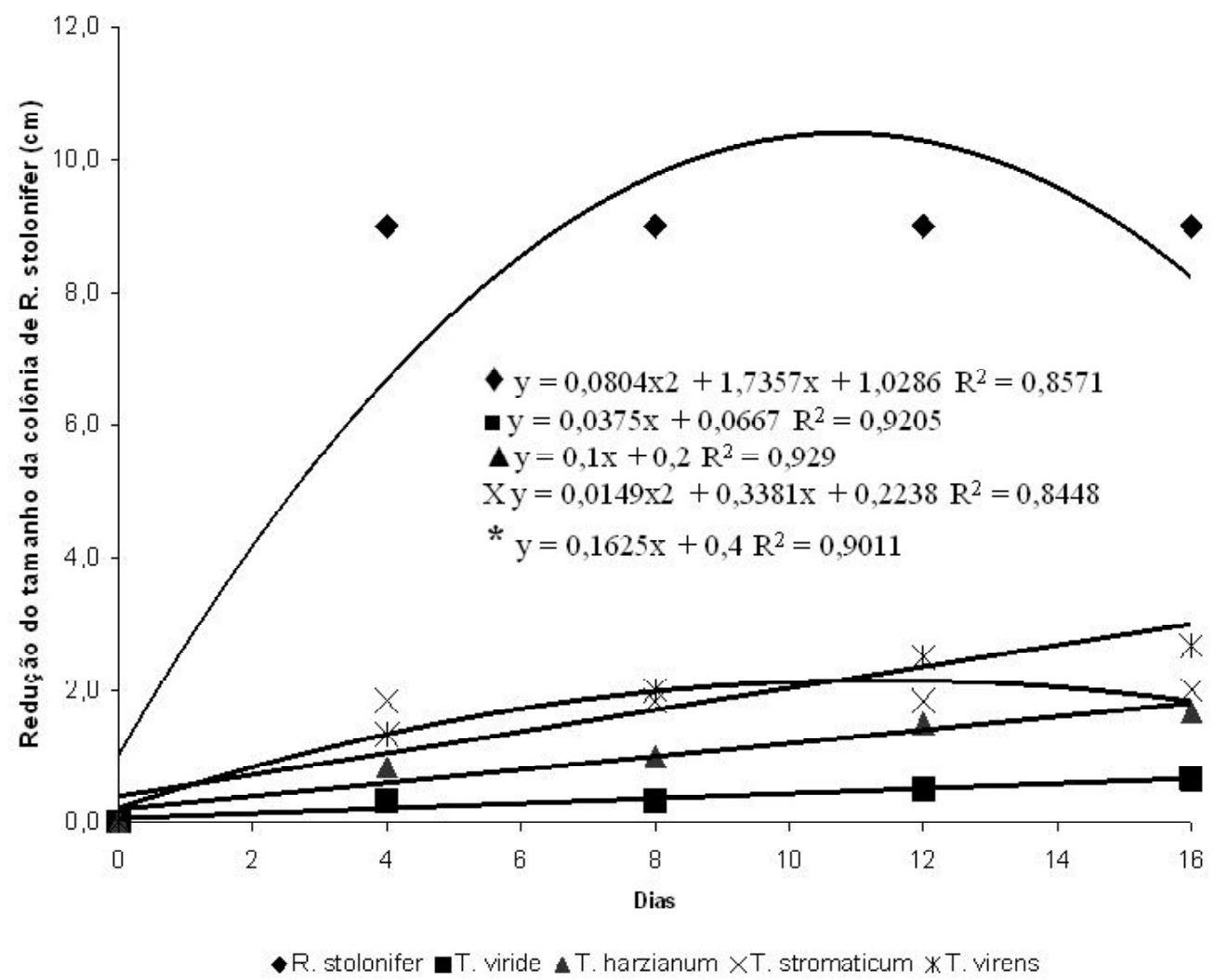

Figura 2. Efeito de metabólitos voláteis produzidos por Trichoderma spp. sobre o crescimento micelial de Rhizopus stolonifer. 


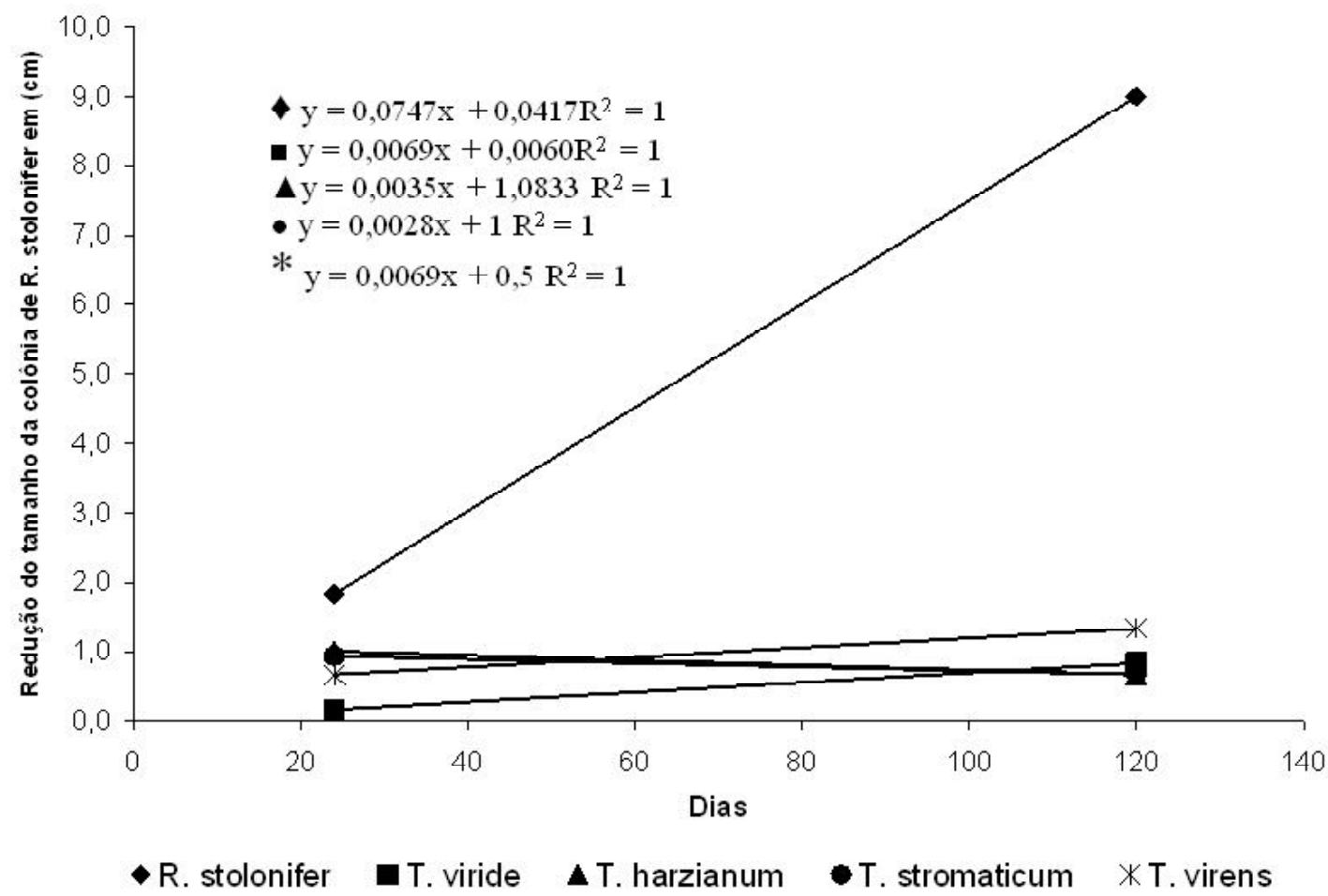

Figura 3 - Avaliação de metabólitos não voláteis produzidos por Trichoderma spp.

\section{Interação in vivo}

$\mathrm{O}$ antagonismo dos isolados de Trichoderma spp. in vitro foi confirmado através dos resultados obtidos em campo. Quando comparado os dados com a testemunha (Tabela 3), constatou-se um controle eficiente do fitopatógeno por todas as espécies de Trichoderma estudadas.

Os dados revelam que a doença ocorreu com grande intensidade e que, houve efeito significativo dos tratamentos aplicados. O Número Médio de Frutos Efetivos (NMFE) entre os antagonistas em estudo foi de $74 \%$ para os tratamentos $\mathrm{T}_{3}$ e $\mathrm{T}_{5}$ e de $75 \%$ para os tratamentos $\mathrm{T}_{2}$ e $\mathrm{T}_{4}$ enquanto que a testemunha teve um percentual de $42 \%$ de vingamento.

Os resultados obtidos no presente trabalho estão de acordo com aqueles encontrados por Ataíde et al., (2) quando estudou o antagonismo de Trichoderma sp. a $R$. stolonifer em cultivo in vivo, obtendo um percentual de vingamento de botões florais de $70 \%$ contra $35 \%$ da testemunha.

O controle biológico, através de introdução de antagonistas, pode ser considerado uma alternativa viável para o controle de enfermidades no campo e na pós-colheita com base em estudos in vitro e in vivo. No controle de doenças da parte aérea espécies de Trichoderma têm

Tabela 3. Efeito dos agentes de controle biológico na porcentagem do (NMFE) número médio de frutos efetivos em condições de campo.

\begin{tabular}{ll}
\hline Tratamentos & NMFE $(\%)$ \\
\hline $\mathrm{T}_{1}-$ Testemunha & $42 \mathrm{~b}$ \\
$\mathrm{~T}_{2}-$ Trichoderma viride & $75 \mathrm{a}$ \\
$\mathrm{T}_{3}-$ Trichoderma harzianum & $74 \mathrm{a}$ \\
$\mathrm{T}_{4}-$ Trichoderma stromaticum & $75 \mathrm{a}$ \\
$T_{5}-$ Trichoderma virens & $74 \mathrm{a}$ \\
\hline
\end{tabular}

Medias seguidas pela mesma letra não difere entre si pelo teste Tukey a 5\% de probabilidade. demonstrado serem efetivas reduzindo até $84 \%$ dos danos causados por Botrytis cinerea e $75 \%$ por Curvularia eragrostidis $(6,12)$.

\section{REFERÊNCIAS BIBLIOGRÁFICAS}

1. Ataide, E. M. Indução floral e produtividade do maracujazeiro-amarelo em função do uso de reguladores de crescimento vegetal. 2005. 100p. Tese (Doutorado) - UNESP Jaboticabal, SP.

2. Ataide, E. M. Controle biológico da podridão floral do maracujá amarelo. Summa Phytopatologica, v. 29. n. 1, p. 96, 2003.

3. Carabi-Adell. Uma técnica simples para observação microscópica de Trichoderma spp. (Hypocreales) em cultura de lâminas. In: Reunião Atual do Instituto Biológico, 15. Arquivos do Instituto Biológico, v. 70 Suplemento, p. 35-37, 2003.

4. Dennis, C.; Webster, J. Antagonistic properties of species groups of Trichoderma. 1- Production of non volatile metabolites. Transactions of the British Mycological Society, London, v. 57, p. 25-39. feb. 1971a.

5. Dennis, C.; Webster, J. Antagonistic properties of species groups of Trichoderma. 3- Hiphal Interaction. Transactions of the British Mycological Society, London, v.57, p.363-369, dec. $1971 \mathrm{~b}$

6. Elad, Y. Shtienberg, D. Integrated management of foliar diseases in greenhouse vegetables according to principles of a decision support systemgreenman. IOBC/WPRS Bulletin, 20, 71-76. 1997.

7. FNP Consultoria \& Agroinformativos. Agrianual 2003: anuário estatístico da agricultura brasileira. São Paulo: 2003. p. 399-405.

8. Goes, A. Doenças fúngicas da parte aérea da cultura do maracujá. In: Simpósio Brasileiro Sobre a Cultura do Maracujazeiro, 5., Jaboticabal. Anais... p. 214-215. 1998.

9. Jackisch-Matsuura, A., Menezes, M. Efeito de Trichoderma spp. no controle de Pythium aphanidermatum em fumo (Nicotiana tabacum ). Summa Phytopathologica, Jaboticabal, v. 25, n. 2, p.161-164, 1999. 
10. Jaill, C.; Apablaza, G.; Noreiro, A. Efecto de la temperatura sobre el crecimiento micelial de Trichoderma harzinum T-39 y la relación interbiótica con Botrytis cinera procedente de tomate. Facultad de Agronomia, pontificia Universidad Católica de Chile, santiago. Disponible en http//alerce.inia.cl/sochifit/ VI.htm\#Articulo_42. Consultado 10 de outubro 2006.

11. Lilly, G.v., Barnett, H.L. Physiology of the fungi. New York: McGraw-Hill Book, 1951. 464p.

12. Michereff, S. J., Menezes, M., Mariano, R. L. R. Antagonismo de espécies de Trichoderma sobre Colletotrichum graminicola, agente da antracnose de sorgo em condições de laboratório. Summa Phytopathologica, Jaguariúna, v. 19, n. 1, p. 14-17, 1993.

13. Ruggiero, C. Maracujá para exportação: aspectos técnicos da produção. Brasília: Embrapa; SPI, 1996. 64p.
14. Ruggiero, C.; Oliveira, J.C. Enxertia do maracujazeiro. In: Simpósio Brasileiro Sobre a Cultura do Maracujá, 5., Jaboticabal, 1998. Anais... Jaboticabal: FUNEP, 1998. p.70-92.

15. São José, A. R.; Rebouças, T. H.H.; Nieto Ange, D.; Pires, M. M. De. Bomfim, P. M. Maracujá práticas de cultivo e comercialização, Vitória da Conquista, BA, UESB/DFZ, 2000. 61p.

16. Tamimi, K. M.; Hutchinson, S. A. Differences between the biological effects of culture gases from several species of Trichoderma. Transactions of the British Mycological Society, London, v. 64, p. 455-463, 1975.

17. Weindling, R. Studies on a lethal principle effective in the parasitic action of Trichoderma lignorum on Rhizoctonia solani and other soil fungi. Phytopathology, v. 24, p. 1140 1153,1964 